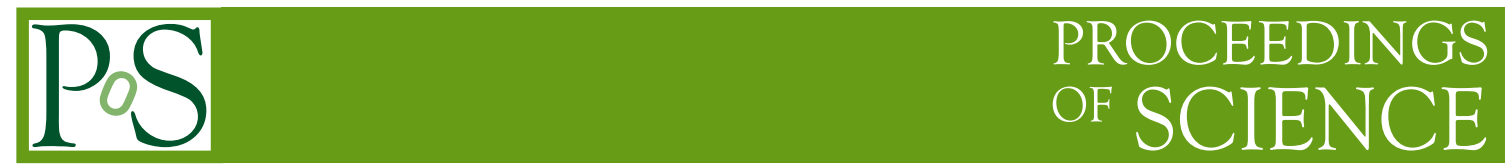

\title{
Neutrino physics outlook
}

\author{
José W. F. Valle * \\ AHEP Group, Institut de Física Corpuscular-C.S.I.C. \\ Universitat de València, Parc Cientific de Paterna. \\ C/Catedratico José Beltrán, 2 E-46980 Paterna (València) - SPAIN \\ E-mail: valledific.uv.es
}

Here I identify some of the opportunities in particle physics associated to the lepton sector, according to their perceived significance, and taking into account the current state-of-the-art.

The 21st international workshop on neutrinos from accelerators (NuFact2019)

August 26 - August 31, 2019

Daegu, Korea

${ }^{*}$ This work was supported by the Spanish grants SEV-2014-0398 and FPA2017-85216-P (AEI/FEDER, UE), PROMETEO/2018/165 (Generalitat Valenciana) and the Spanish Red Consolider MultiDark FPA2017-90566-REDC. 


\section{Neutrino oscillations}

My assigned task was to give an outlook on the field. I will take the opportunity to discuss some of the most interesting directions for future work. These are personal assessments on the most promising opportunities that may be lurking ahead of us. In order to perform my task I start with a "drone view" of the current status of neutrino oscillations. Here the major highlight has been the discovery of the phenomenon, made in solar and atmospheric studies, followed by reactor and accelerator-based experiments that have not only provided independent confirmation, but also improved parameter determination. The current experimental data mainly converge to a consistent global picture in which the oscillation parameters are determined as shown in Fig. 1.
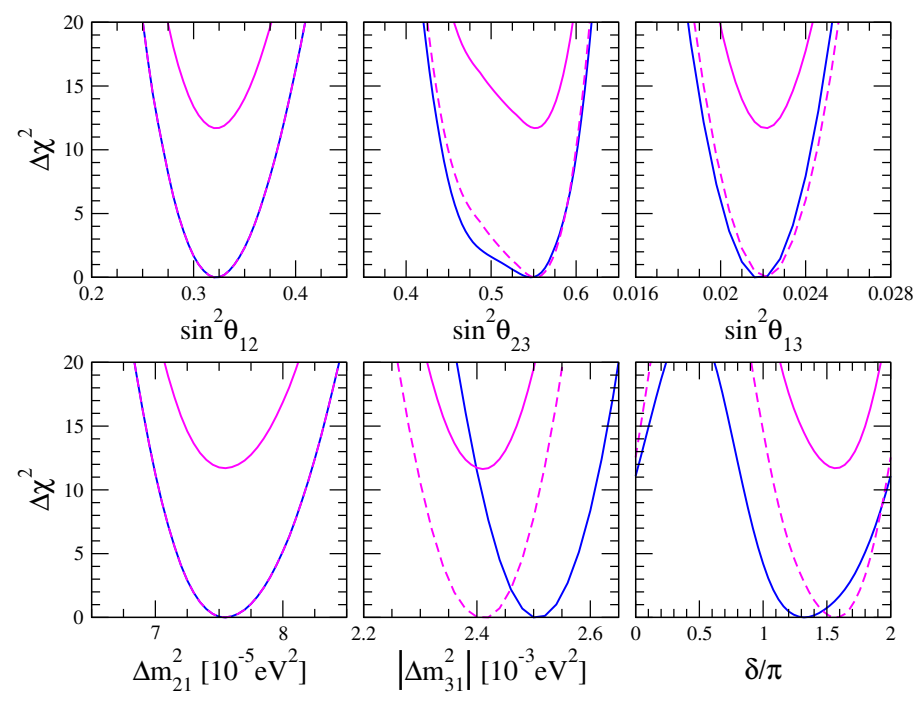

Figure 1: Summary of neutrino oscillation parameters, 2018. From [1].

The top-three panels show the determination of the three mixing angles, where one sees that two of them are large, at odds with the corresponding mixing angles observed in the quark sector. In fact, it is the smaller of the lepton mixing angles the one that lies intriguingly close in magnitude to the largest of the quark mixing angles, namely the Cabibbo angle. Perhaps nature tries to tell us a secret right there. One also sees that, although the overall picture of such "three-neutrino paradigm" is rather appealing, there are still some loose ends. The normal-ordered neutrino spectrum is preferred by slightly more than $3 \sigma$, while the octant of the atmospheric angle and the value of the leptonic CP phase are still poorly determined.

The ordering of the neutrino mass spectrum, as well as CP determination and octant resolution remain open challenges that form the target of the next generation of oscillation searches. $\mathrm{CP}$ determination will be the task of the Deep Underground Neutrino Experiment (DUNE). The experiment will consist of two detector systems placed along Fermilab's Long Baseline Neutrino Facility (LBNF) beam [2]. One detector system will be near the beam source, while a much larger one will be placed underground $1300 \mathrm{~km}$ away at the Sanford Underground Research Laboratory in South Dakota, in the same mine where Ray Davis has pioneered our field in the sixties.

The situation concerning the CP phase is illustrated in Fig. 2. The current status of CP is shown in blue in the left panel, as determined from the Valencia global fit (there is very good 

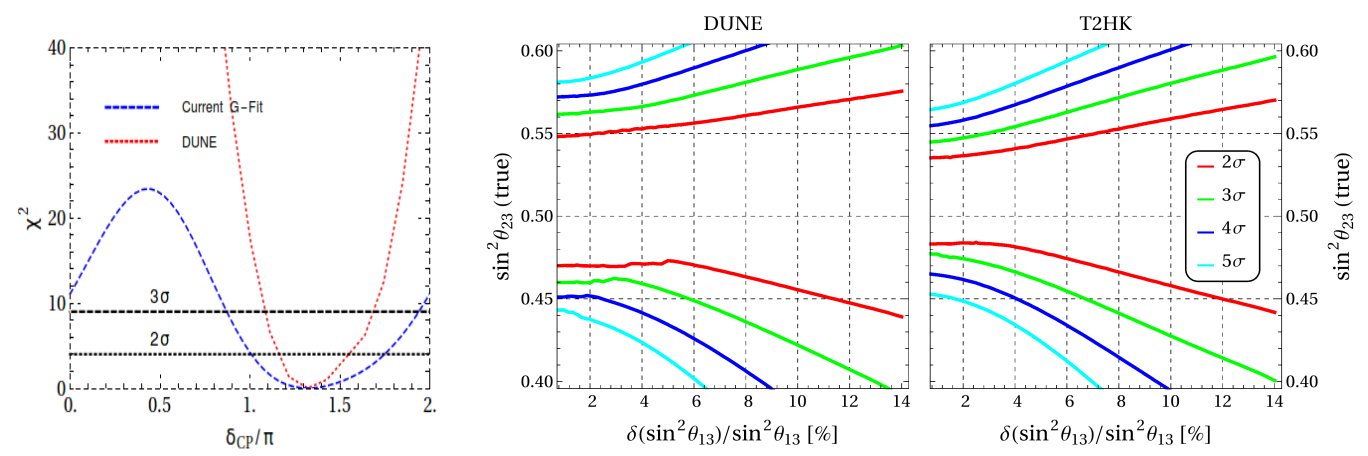

Figure 2: Prospects for CP measurement and octant determination. From [3, 4].

agreement amongst the three global fitting groups), while the future prospects are shown in red. The latter assume that the best fit value of the phase remains as given now, and that DUNE performs according to design. In this case the experiment will suffice to establish CP violation within the three-neutrino paradigm. In contrast, the other panels show that the octant resolution will not be fully achieved even by the ambitious T2HK proposal in Japan. See Ref. [3, 4, 5] for details and related discussions.

Nevertheless, the two decades that followed the oscillation discovery have brought a tremendous progress in the determination of the pattern of leptonic mixing! Its most salient features are nicely captured by the tri-bimaximal (TBM) mixing ansatz [6]. However, the TBM predictions are now at odds with observations from reactor experiments [7], and with $\mathrm{CP}$ violation hints from long-baseline oscillation experiments, such as T2K [8]. Fortunately, there are systematic ways of generalizing patterns with manifest $\mu \tau$ reflection symmetry, such as TBM. These "revamped" patterns are obtained by exploiting partially conserved generalized CP symmetries [9, 10, 11], and their predictions may be tested with precision studies at DUNE [3]. Indeed, as an example, the CP phase can be predicted to lie along the green band in Fig. 3 [12]. One sees how the overlap of the predicted band with the 3-neutrino global oscillation region selects a narrow allowed range for $\delta_{C P}$ in the upper branch. Note however that, at least in this particular case, the octant determination challenge remains pretty much unresolved.

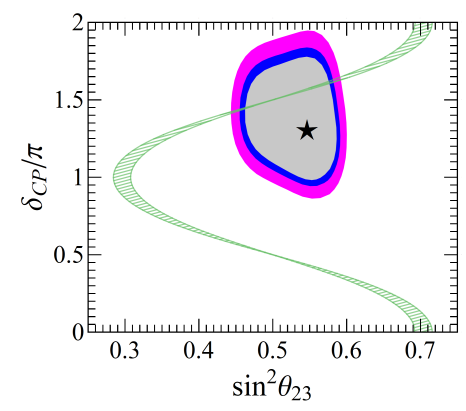

Figure 3: Predicting the CP phase in a realistic generalized TBM scenario, from [12].

There are other alternatives to the TBM pattern of neutrino mixing, for example bi-large mixing schemes $[13,14]$. These exploit the observation made above that the largest quark-mixing is similar in magnitude to the smallest of the lepton mixing angles, suggesting that the Cabibbo angle 
may be taken as the universal seed for flavor mixing [15]. Remarkably, in such "bi-large" lepton mixing schemes the good measurement of $\theta_{13}$ at reactor experiments allows one to predict both solar and atmospheric mixing angles [16] pretty well, as seen in Fig. 4, left panel. This also leads to sharp predictions for the $\mathrm{CP}$ phase, up to the degeneracy seen in the right panel, where two symmetric branches can be appreciated.
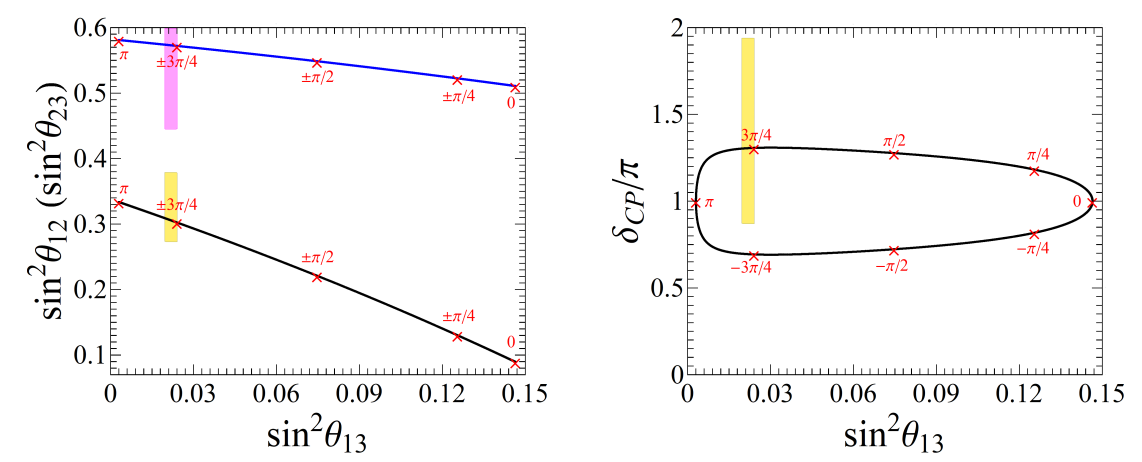

Figure 4: Predictions for mixing angles and CP violation in the "bi-large" scheme of Ref. [16].

There are also "softer" bi-large-type lepton mixing patterns in which the oscillation parameters are expressed in terms of two independent parameters, $\phi$ and $\psi$, as seen in Fig. 5. One sees how in this case the individual determinations of the three angles and the phase nicely converge into a unique region in the upper plane, indicating the utility of the bi-large description.

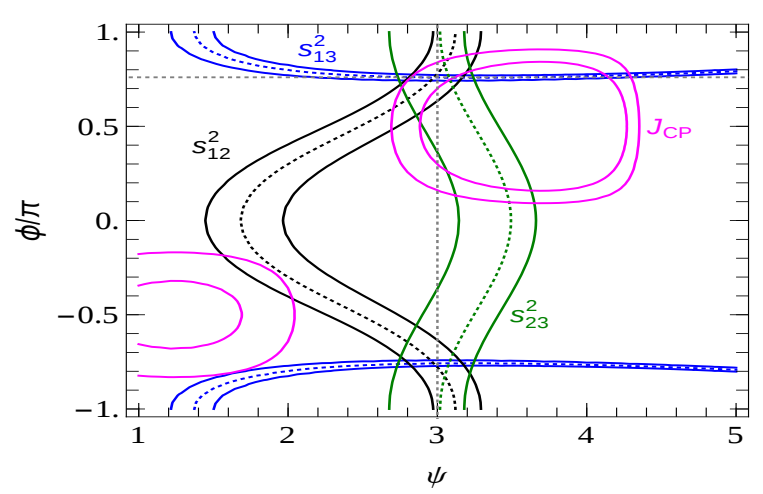

Figure 5: Predictions for mixing angles and CP violation in a generalized "bi-large" scheme, from [17].

This brings us now to discuss the issue of robustness of the oscillation interpretation of neutrino data. If neutrinos acquire mass from the exchange of heavy neutral isosinglet lepton mediators, as in the simplest seesaw paradigm, then these (mass-eigenstates) will couple (sub-dominantly) by mixing in the leptonic charged current [18]. Since these states are too heavy to take part in oscillations, the mixing matrix describing the propagation of the three light neutrinos will not be strictly unitary $[19,20,21,22,23]$. The effective $3 \times 3$ mixing matrix will be written as,

$$
K_{L}=N^{N P} U
$$


where the unitary matrix $U$ is pre-multiplied by a triangular matrix given as

$$
N^{N P}=\left(\begin{array}{ccc}
\alpha_{11} & 0 & 0 \\
\alpha_{21} & \alpha_{22} & 0 \\
\alpha_{31} & \alpha_{32} & \alpha_{33}
\end{array}\right)
$$

Its diagonal elements are real and close to one, while the off-diagonals are small but complex. It has been shown that the phase in $\alpha_{21}$ plays a crucial role in the CP measurement at DUNE. Indeed, there is an intrinsic ambiguity in probing $\mathrm{CP}$ violation in neutrino oscillations, arising from the confusion between the phase in $\alpha_{21}$ with that which characterizes the three-neutrino paradigm [24]. For example, for $\mathrm{L} / \mathrm{E}=500 \mathrm{~km} / \mathrm{GeV}$ the vacuum appearance probability exhibits a degeneracy for different phase combinations. This shows up in the $P_{\mu e}$ isocontours as a function of the two CP phases, as indicated in Fig. 6. The solid line corresponds to the standard value $P_{\mu e}$ with $\delta_{C P}=3 \pi / 2$, while colored regions denote the corresponding $10 \%$ and $20 \%$ deviations.

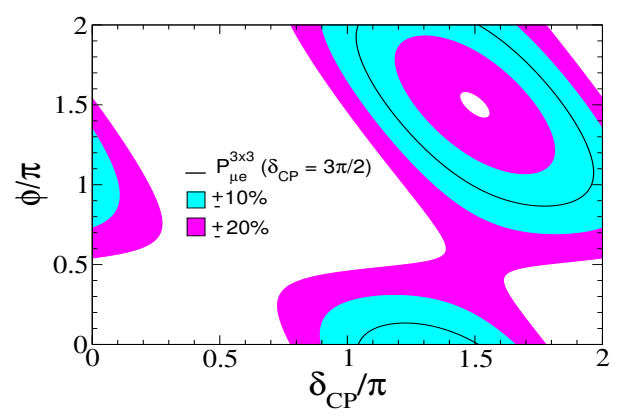

Figure 6: New ambiguity in probing CP violation in neutrino oscillations, from Ref. [24].

There have been suggestions to mitigate this problem [25], whose main ingredient is near detection of neutrinos, capable of improving the limits on the magnitude of $\alpha_{21}$. The current bounds on the latter lie at the per-cent level and have been carefully compiled in Ref. [26], stressing the robustness of neutrino-only limits. The short-baseline neutrino program at Fermilab is ideally suited to probe the unitarity of the lepton mixing matrix, with meaningful sensitivities, potentially better than existing bounds arising from current neutrino experiments [27], as illustrated in Fig. 7.

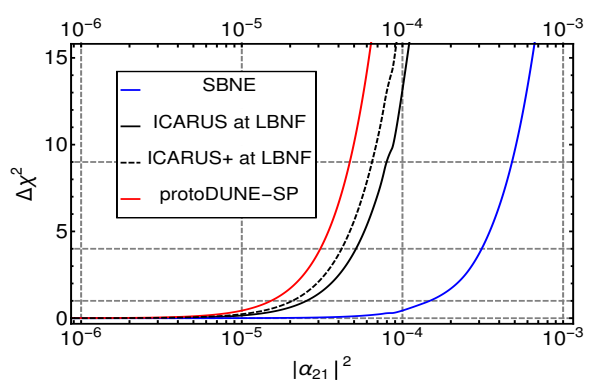

Figure 7: Improved leptonic unitarity tests at various Fermilab near-distance setups, from Ref. [27].

It would be desirable to perform more dedicated experimental studies of the potential of various near-distance setups, taking into account their practical feasibility. 
Needless to say that unitarity violation is only the simplest example of non-standard neutrino interactions (NSI). These are a generic feature of low-scale models of neutrino mass generation [28], and their study constitutes an important topic in neutrino physics [29] as it could shed light on the scale of neutrino mass generation and probe the robustness of the neutrino oscillation interpretation [30]. NSI could also bring degeneracies in the determination of the CP phase, similar to what we just discussed [31]. As a result, NSI studies constitute a necessary topic in the agenda of upcoming oscillation experiments [32].

\section{Absolute neutrino mass}

Although the oscillation programme has driven much of the "revolution" our field has experienced over the last few decades, by themselves, oscillation studies are insensitive to the absolute scale of neutrino mass. Single and double beta decay processes (as well as cosmology [33]) can probe the absolute neutrino mass in complementary ways.

Recently the Katrin experiment has derived an upper limit of $1.1 \mathrm{eV}$ (at $90 \%$ C.L.) on the absolute mass scale of neutrinos [34] from the study of Tritium endpoint spectrum. This bound applies irrespectively of whether neutrinos are Dirac or Majorana particles. In the latter case one expects also a neutrinoless variety of double beta decay - dubbed $0 v \beta \beta$ for short - in which no neutrinos are emitted as real particles. Rather, the process involves the virtual lepton number violating propagation of neutrinos.
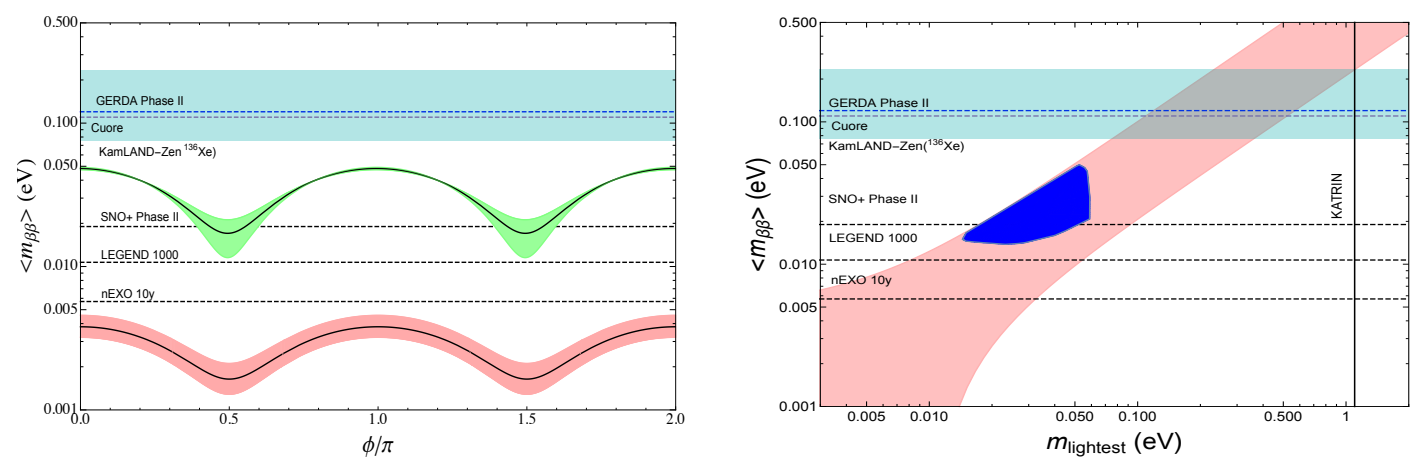

Figure 8: Lower bound on the $0 v \beta \beta$ decay amplitude in a two-massive-Majorana-neutrino model, e.g. [35], (left), and in the flavor-symmetric three-massive-Majorana-neutrino model of [36] (right).

One can determine the expected ranges for the decay amplitude, taking into account the allowed neutrino oscillation parameters given above. One finds that for inverted-ordered neutrino masses, there is a lower bound for the $0 v \beta \beta$ amplitude, while for normal-ordered neutrinos there is none, indicating the possibility of having full destructive interference amongst the three light neutrinos. This is of course discouraging for experiment.

However, if one of the three neutrinos is massless or nearly so, there is no cancellation, even if neutrinos are normal-ordered. Note that the resulting lower bound correlates with the only free parameter, the relative Majorana phase between the two neutrinos, see left panel in Fig. 8. As a result, the allowed $0 v \beta \beta$ ranges are also much narrower than in the generic three-neutrino case. This situation emerges in a number of theories, such as the original missing partner seesaw mechanism [18] and many other schemes, for example the one considered in [35]. 
Even if all three neutrinos are massive, it could happen that cancellation is prevented by the flavor structure of the leptonic weak interaction vertex, as predicted by a number of flavor symmetries [37, 38]. Either way, there is hope that upcoming experiments may shed light on the relevant Majorana phase, as indicated by the estimated experimental sensitivities [39, 40, 41, 42, 43, 44, 45]. For an alternative recent compilation of $0 v \beta \beta$ sensitivities see [46].

To sum up the $0 v \beta \beta$ discussion, we now recall the old black-box theorem [47], revisited in Ref. [48]. That argument captures the significance of the $0 v \beta \beta$ decay by stating that, if this process is ever discovered, it would imply the Majorana nature of (at least one of) the neutrinos. The conclusion holds irrespective of whether $0 v \beta \beta$ arises from neutrino exchange or from a shortrange mechanism that might be probed at colliders, such as the LHC.

\section{Origin of neutrino mass}

Forty years ago Weinberg noted that, even though the Standard Model lacks neutrino masses, one can induce them through a unique dimension five operator [49] associated to new physics with lepton number non-conservation. The most popular "UV-completion" of the dimension five operator is provided by the seesaw mechanism. Here neutrino masses are induced by the exchange of heavy fermions (type I) or scalars (type II), a terminology which is opposite to the one adopted in [18]. The new seesaw states were originally thought to lie at a high mass scale, associated to unification, whose most characteristic predictions (proton decay) have so far not been vindicated.

Here we focus instead on "low-scale" realizations of the seesaw mechanism [50, 51, 52, 53]. Notice that, at the standard $\mathrm{SU}(3)_{\mathrm{c}} \otimes \mathrm{SU}(2)_{\mathrm{L}} \otimes \mathrm{U}(1)_{\mathrm{Y}}$ level, "right-handed" neutrinos are gaugesinglets, so their number is arbitrary, it need not match the number of "left-handed" ones [18].

Hence one can add less "right-" than "left-handed" neutrinos. As an example, one may consider a $(3,2)$ seesaw scheme, with three "left-" and only two "right" neutrinos. In this case one of the "left-handed" neutrinos remains "unpaired" and hence massless [18]. In such "missing partner seesaw" both solar and atmospheric scales arise from the seesaw mechanism, and the lightest neutrino remains massless, leading to the $0 v \beta \beta$ lower bound we just discussed.

Likewise, a $(3,1)$ seesaw scheme can be envisaged. In this case only one mass scale is generated by the tree-level seesaw mechanism, while the other, the solar scale, may arise from some loop mechanism mediated, for example, by supersymmetry $[54,55]$, or by a "dark sector", as in the simplest scotogenic seesaw mechanism [56].

Alternatively, one may add more "right-" than "left-handed" neutrinos. As a very interesting example, one can add two isosinglets per family of leptons, sequentially. By imposing lepton number conservation on such $(3,6)$ scheme (again, in the same notation of [18]) one gets a "template" scheme with massless neutrinos, exactly as in the Standard Model. In other words, such simple setup leads to massless neutrinos within perturbation theory, as long as lepton number symmetry is exact. In contrast to the Standard Model case, however, lepton flavor is violated, and similarly, CP symmetry. This has two important implications. First, it elucidates the meaning of flavor and CP violation in the leptonic weak interaction, implying that such processes need not be suppressed by the smallness of neutrino masses, and can therefore be large [57, 58, 59, 60, 61]. Second, this reference model serves also as template for building genuine low-scale seesaw schemes where neutrino masses are protected by lepton number symmetry [50, 51, 52, 53]. 
In short, neutrino masses are naturally small as a result of symmetry protection both in the high-scale as well as low-scale seesaw approaches. One expects a rich phenomenology in lowscale seesaw, in contrast to the high-scale seesaw mechanism.

We now turn to the possibility of Dirac neutrinos. Whether or not neutrinos are Dirac-type is an experimental open question. If neutrinos happen to be Dirac particles, symmetry is required not only to account for the small neutrino masses, but also to ensure Diracness. In fact, there might be a deep reason for neutrinos to be Dirac-type. For example, this could be associated to the stability of dark matter [62,63] or to the existence of a Peccei-Quinn symmetry [64]. In the last few years there have been detailed classifications of Dirac seesaw mechanisms $[65,66]$ as well as full-fledged UV-complete model constructions [62, 63, 67, 68, 69, 70].

An important point to note is that the seesaw opens the way for a dynamical understanding of small neutrino masses where, in addition to the standard vacuum expectation value (vev) $v_{2}$ responsible for electroweak breaking, there are new vevs coupled to neutrinos. These include an isotriplet $v_{3}$ coupled to "left-handed" neutrinos, and an isosinglet $v_{1}$ coupled to "right" neutrinos. These may trigger the spontaneous violation of lepton number, accompanied by a Goldstone boson, dubbed Majoron [71]. The dynamical $\mathrm{SU}(3)_{\mathrm{c}} \otimes \mathrm{SU}(2)_{\mathrm{L}} \otimes \mathrm{U}(1)_{\mathrm{Y}}$ seesaw mechanism with hierarchical vevs $v_{1} \gg v_{2} \gg v_{3}$ was proposed in [72]. It has been noted that the extra vevs can substantially affect and improve vacuum stability and perturbative unitarity within low-scale seesaw schemes [73, 74].

\section{Probing the mediators of neutrino mass generation}

For definiteness, here we take the simplest low-scale type-I seesaw mechanism of neutrino mass generation $[50,51,52,53]$. As we saw, if the seesaw mediators lie in the TeV scale, they can lead to a variety of observable effects. For example, their existence will affect the description of neutrino oscillations, by having an effectively non-unitary mixing matrix describing the propagation of the three light neutrinos [19, 20,21, 22, 23]. As we have already discussed, this brings in extra CP violation that can fake the one expected within the simplest three-neutrino paradigm [24]. As a result, unitarity violation degrades the CP violation sensitivity expected at DUNE [26].

Low-scale seesaw models also open the possibility of direct production of the mediators of neutrino mass generation in collider experiments. Since the neutrino mass mediators in type-I seesaw are gauge singlets, they couple to the weak currents only through mixing [18]. Yet, already in the pre-LEP days, it was suggested that the isosinglet heavy leptons $N$ present in type-I seesaw would be singly-produced in $Z$ boson decays, as $Z \rightarrow N+v$, where $v$ is a light neutrino [75]. Given the large number of $Z$ 's at the peak, the detection of the associated signatures would be feasible all the way up to masses close to the $\mathrm{Z}$ mass [75]. The Delphi and L3 collaborations have later performed this search at the Z-peak and above. In the latter case one covers a wider kinematical range, but the sensitivity worsens due to the lower rates.

Proton-proton collisions at the LHC would singly-produce the $N$ by a Drell-Yan-type mechanism, and searches have been performed by ATLAS and CMS, further extending the kinematical reach. Again, as a result of the low single-production rates, the sensitivity is not as high.

A way to avoid the mixing suppression is to imagine the existence of a production portal provided by new vector bosons associated to an extended gauge symmetry [76, 77, 78]. In this case, the exchange of a new $Z^{\prime}$ can pair-produce the $N$ a la Drell-Yan, leading to improved sensitivi- 
ties $[76,77,78]$. Most interestingly, each $N$ can decay to any flavor of charged leptons. This would lead to a unique possibility of charged lepton flavor violation at high energies, proposed in [57]. Moreover, since the $N$ decays through mixing, the associated events may show a displaced vertex, as seen in Fig. 9, right panel.
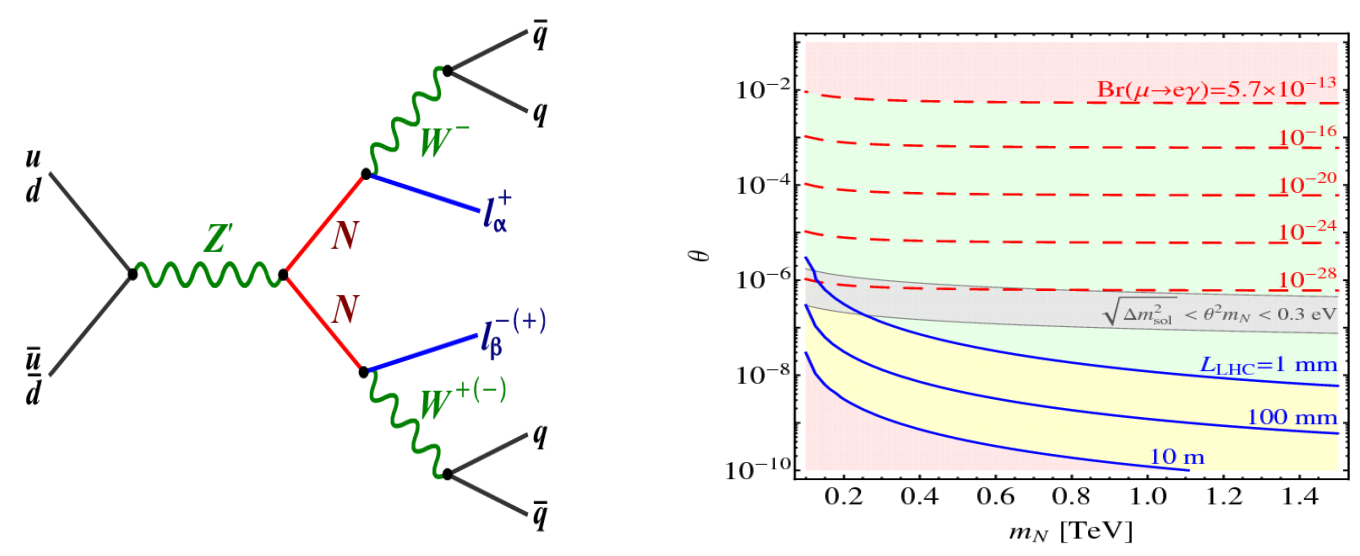

Figure 9: Average decay length of a heavy neutrino $N$ produced via a $Z^{\prime}$ decaying as $Z^{\prime} \rightarrow N N$, versus the mass $m_{N}$ and the light-heavy mixing angle $\theta$ (solid blue contours), from [77], see text.

The plot shows the average decay length of a heavy neutrino $N$ produced via a $3 \mathrm{TeV}$ mass $Z^{\prime}$ decaying as $Z^{\prime} \rightarrow N N$, versus $m_{N}$ and the light-heavy mixing angle $\theta$ (solid blue contours). The dashed-red contours denote constant values for $B R(\mu \rightarrow e+\gamma)$, whereas the grey shaded band corresponds to parameter values which produce light neutrino mass scales around $0.3 \mathrm{eV}$ within the simplest type-I seesaw mechanism. One sees that, for some parameter values, the sensitivity attainable at high energies will exceed that of low-energy lepton flavor violation processes such as $\mu \rightarrow e+\gamma$. This nicely illustrates the complementarity between high energy colliders and the high intensity approach pursued at muon facilities. Moreover, it provides a real possibility for an important (double) discovery at the LHC, i.e. that of the mediator of neutrino mass generation, as well as the discovery of the charged lepton flavor violation phenomenon at high energies!

\section{Conclusion}

All in all, the legacy of the oscillation programme over the last two decades has been a tremendous progress in our field, bringing neutrinos to the center of the particle physics stage. Indeed, addressing the dynamical origin of small neutrino masses touches the heart of the electroweak theory, such as the consistency of symmetry breaking. Besides neutrino mass dynamics, there are other issues in particle physics and cosmology for which neutrinos may provide key input. For example, they could shed light into the flavor problem [79, 80, 81, 82], give us a glimpse for the existence of extra dimensions [82], or suggest new pathways to unification [83, 84]. Perhaps they could also make a step forward towards the final dream of unifying the existing forces and the observed particle families together [85]. Last but not least, dark matter and neutrinos could be intimately related, so neutrinos may, in some sense, also hold the key to the solution of the cosmological dark matter problem. For example, dark matter could be the mediator of neutrino mass 
generation [86, 87]. Unfortunately, I will not have the space to go into these in detail, see [88] for some comments.

From the experimental viewpoint in the coming decade we expect a vibrant period for oscillations studies, within and beyond the minimum paradigm. Studies should also be performed using neutral current phenomena. Likewise $0 v \beta \beta$ searches may lead to a breakthrough in the next decade or so. High energy studies at the LHC may prove better for charged lepton flavor violation searches than the conventional high intensity muon facilities, and might have a chance to actually discover the mediators ultimately responsible for neutrino mass generation!

I take the opportunity to thank all of my collaborators for the good moments we have shared doing neutrino physics. In particular I thank O. Miranda for going through the text.

\section{References}

[1] P.F. de Salas et al. Status of neutrino oscillations 2018: $3 \sigma$ hint for normal mass ordering and improved CP sensitivity. Phys.Lett., B782:633-640, 2018.

[2] R. Acciarri et al. Long-Baseline Neutrino Facility (LBNF) and Deep Underground Neutrino Experiment (DUNE). e-Print 1512.06148 (2015).

[3] Newton Nath, Rahul Srivastava, and José W.F. Valle. Testing generalized CP symmetries with precision studies at DUNE. Phys.Rev., D99:075005, 2019.

[4] Sabya Sachi Chatterjee, Pedro Pasquini, and J.W.F. Valle. Resolving the atmospheric octant by an improved measurement of the reactor angle. Phys.Rev., D96:011303, 2017.

[5] R.Srivastava et al. Zooming in on neutrino oscillations with DUNE. Phys.Rev., D97:095025, 2018.

[6] P. F. Harrison, D. H. Perkins, and W. G. Scott. Tri-bimaximal mixing and the neutrino oscillation data. Phys. Lett., B530:167, 2002.

[7] F.P. An et al. Observation of electron-antineutrino disappearance at Daya Bay. Phys.Rev.Lett., 108:171803, 2012.

[8] K. Abe et al. Observation of Electron Neutrino Appearance in a Muon Neutrino Beam. Phys.Rev.Lett., 112:061802, 2014.

[9] Peng Chen et al. Generalized $\mu-\tau$ reflection symmetry and leptonic CP violation. Phys.Lett., B753:644-652, 2016.

[10] Peng Chen et al. Classifying CP transformations according to their texture zeros: theory and implications. Phys.Rev., D94:033002, 2016.

[11] Peng Chen et al. Neutrino Predictions from Generalized CP Symmetries of Charged Leptons. JHEP, 1807:077, 2018.

[12] Peng Chen et al. Realistic tribimaximal neutrino mixing. Phys.Rev., D98:055019, 2018.

[13] S.M. Boucenna et al. Bi-large neutrino mixing and the Cabibbo angle. Phys.Rev., D86:051301, 2012.

[14] Gui-Jun Ding, S. Morisi, and J.W.F. Valle. Bilarge neutrino mixing and Abelian flavor symmetry. Phys.Rev., D87:053013, 2013.

[15] S. Roy et al. The Cabibbo angle as a universal seed for quark and lepton mixings. Phys.Lett., B748:1-4, 2015. 
[16] Peng Chen et al. Predicting neutrino oscillations with "bi-large" lepton mixing matrices. Phys.Lett., B792:461-464, 2019.

[17] Gui-Jun Ding et al. Status and prospects of 'bi-large' leptonic mixing. Phys.Lett., B796:162, 2019.

[18] J Schechter and J. W. F. Valle. Neutrino Masses in SU(2)xU(1) Theories. Phys. Rev, D22:2227, 1980.

[19] J. W. F. Valle. Resonant Oscillations of Massless Neutrinos in Matter. Phys.Lett., B199:432, 1987.

[20] H. Nunokawa et al. Resonant conversion of massless neutrinos in supernovae. Phys.Rev., D54:4356-4363, 1996.

[21] S. Antusch, C. Biggio, E. Fernandez-Martinez, M.B. Gavela, and J. Lopez-Pavon. Unitarity of the Leptonic Mixing Matrix. JHEP, 0610:084, 2006.

[22] O.G. Miranda and J.W.F. Valle. Neutrino oscillations and the seesaw origin of neutrino mass. Nucl.Phys., B908:436-455, 2016.

[23] F.Escrihuela et al. On the description of non-unitary neutrino mixing. Phys. Rev, D92:053009, 2015.

[24] O.G. Miranda, M. Tortola, and J. W. F. Valle. New ambiguity in probing CP violation in neutrino oscillations. Phys.Rev.Lett., 117:061804, 2016.

[25] Shao-Feng Ge et al. Measuring the leptonic $\mathrm{CP}$ phase in neutrino oscillations with nonunitary mixing. Phys.Rev., D95:033005, 2017.

[26] F. J. Escrihuela et al. Probing CP violation with non-unitary mixing in long-baseline neutrino oscillation experiments: DUNE as a case study. New J.Phys., 19:093005, 2017.

[27] O. G. Miranda, Pedro Pasquini, M. Tórtola, and J. W. F. Valle. Exploring the Potential of Short-Baseline Physics at Fermilab. Phys. Rev., D97(9):095026, 2018.

[28] Sofiane M. Boucenna, Stefano Morisi, and Jose W. F. Valle. The low-scale approach to neutrino masses. Adv. High Energy Phys., 2014:831598, 2014.

[29] M. Maltoni et al. Status of global fits to neutrino oscillations. New J.Phys., 6:122, 2004; J. Kopp, M. Lindner, T. Ota and J. Sato, Non-standard neutrino interactions... Phys. Rev. D77:013007, 2008

[30] O.G. Miranda, M.A. Tortola, and J.W.F. Valle. Are solar neutrino oscillations robust? JHEP, 0610:008, 2006; P. Coloma, P. B. Denton, M. C. Gonzalez-Garcia, M. Maltoni and T. Schwetz, Curtailing the Dark Side in Non-Standard Neutrino Interactions JHEP 1704: 116, 2017

[31] L.J. Flores, E.A. Garcés, and O.G. Miranda. Exploring NSI degeneracies in long-baseline experiments. Phys.Rev., D98:035030, 2018.

[32] P.S. Bhupal Dev et al. Neutrino Non-Standard Interactions: A Status Report. 1907.00991.

[33] M. Lattanzi and M. Gerbino, Status of neutrino properties ... Front in Phys. 5:70, 2018.

[34] M. Aker et al. An improved upper limit on the neutrino mass from a direct kinematic method by KATRIN. Phys. Rev. Lett., 123:221802, Nov 2019.

[35] M. Reig et al. Bound-state dark matter with Majorana neutrinos. Phys.Lett., B790:303-307, 2019.

[36] S.F. King et al. Quark-Lepton Mass Relation in a Realistic $A_{4}$ Extension of the Standard Model. Phys.Lett., B724:68-72, 2013.

[37] L. Dorame et al. Constraining Neutrinoless Double Beta Decay. Nucl.Phys., B861:259-270, 2012.

[38] L. Dorame et al. A new neutrino mass sum rule from inverse seesaw. Phys.Rev., D86:056001, 2012. 
[39] A. Gando et al. Search for Majorana Neutrinos near the Inverted Mass Hierarchy Region with KamLAND-Zen. Phys.Rev.Lett., 117:082503, 2016.

[40] C. Alduino et al. First Results from CUORE: A Search for Lepton Number Violation via $0 v \beta \beta$ Decay of ${ }^{130}$ Te. Phys.Rev.Lett., 120:132501, 2018.

[41] J.B. Albert et al. Search for Neutrinoless Double-Beta Decay with the Upgraded EXO-200 Detector. Phys.Rev.Lett., 120:072701, 2018.

[42] M. Agostini et al. Improved Limit on Neutrinoless Double- $\beta$ Decay of ${ }^{76}$ Ge from GERDA Phase II. Phys.Rev.Lett., 120:132503, 2018.

[43] S. Andringa et al. Current Status and Future Prospects of the SNO+ Experiment. Adv.High Energy Phys., 2016:6194250, 2016.

[44] N. Abgrall et al. The Large Enriched Germanium Experiment for Neutrinoless Double Beta Decay (LEGEND). volume 1894, page 020027, 2017.

[45] J.B. Albert et al. Sensitivity and Discovery Potential of nEXO to Neutrinoless Double Beta Decay. Phys.Rev., C97:065503, 2018.

[46] M. Agostini et al. Probing Majorana neutrinos with double- $\beta$ decay. Science, 365:1445, 2019.

[47] J. Schechter and J.W.F. Valle. Neutrinoless Double beta Decay in SU(2) x U(1) Theories. Phys.Rev., D25:2951, 1982.

[48] Michael Duerr, Manfred Lindner, and Alexander Merle. On the Quantitative Impact of the Schechter-Valle Theorem. JHEP, 1106:091, 2011.

[49] Steven Weinberg. Baryon and Lepton Nonconserving Processes. Phys.Rev.Lett., 43:1566-1570, 1979.

[50] R.N. Mohapatra and J.W.F. Valle. Neutrino Mass and Baryon Number Nonconservation in Superstring Models. Phys.Rev., D34:1642, 1986.

[51] Evgeny Akhmedov et al. Dynamical left-right symmetry breaking. Phys.Rev., D53:2752-2780, 1996.

[52] Evgeny Akhmedov et al. Left-right symmetry breaking in NJL approach. Phys.Lett., B368:270, 1996.

[53] Michal Malinsky, J.C. Romao, and J.W.F. Valle. Novel supersymmetric SO(10) seesaw mechanism. Phys.Rev.Lett., 95:161801, 2005.

[54] M. Hirsch et al. Neutrino masses and mixings from supersymmetry with bilinear R parity violation: A Theory for solar and atmospheric neutrino oscillations. Phys.Rev., D62:113008, 2000.

[55] M.A. Diaz et al. Solar neutrino masses and mixing from bilinear R parity broken supersymmetry: Analytical versus numerical results. Phys.Rev., D68:013009, 2003.

[56] N.Rojas, R.Srivastava, J.Valle. Simplest Scoto-Seesaw Mechanism. Phys.Lett, B789:132, 2019.

[57] J. Bernabeu et al. Lepton Flavor Nonconservation at High-Energies in a Superstring Inspired Standard Model. Phys.Lett., B187:303, 1987.

[58] G.C. Branco, M.N. Rebelo, and J.W.F. Valle. Leptonic CP Violation with Massless Neutrinos. Phys.Lett., B225:385, 1989.

[59] N.Rius and J. Valle. Leptonic CP Violating Asymmetries in Z Decays. Phys.Lett, B246:249, 1990.

[60] F. Deppisch and J.W.F. Valle. Enhanced lepton flavor violation in the supersymmetric inverse seesaw model. Phys.Rev., D72:036001, 2005.

[61] Deppisch et al. Enhanced $\mu$ e conversion in nuclei in the inverse seesaw. Nucl.Phys., B752:80, 2006. 
[62] S.Centelles et al, Dirac Neutrinos and Dark Matter Stability... Phys.Lett, B767:209, 2017.

[63] S. Centelles et al. Generalized bottom-tau unification, neutrino oscillations Phys.Lett, B773:26, 2017.

[64] E. Peinado et al. Dirac neutrinos from Peccei-Quinn symmetry: a fresh look at the axion. 1910.02961.

[65] S.Centelles et al Seesaw roadmap to neutrino mass and dark matter. Phys. Lett., B781:122-128, 2018.

[66] S.Centelles et al, Seesaw Dirac neutrino mass through dimension-six operators. Phys.Rev, D98:035009, 2018.

[67] Mario Reig, Jose W. F. Valle, and C.A. Vaquera-Araujo. Realistic $\mathrm{SU}(3)_{\mathrm{c}} \otimes \mathrm{SU}(3)_{\mathrm{L}} \otimes \mathrm{U}(1)_{\mathrm{X}}$ model with a type II Dirac neutrino seesaw mechanism. Phys.Rev., D94:033012, 2016.

[68] J.Valle, C.Vaquera. Dynamical seesaw mechanism for Dirac neutrinos. Phys.Lett, B755:363, 2016.

[69] C.Bonilla, and J.Valle. Naturally light neutrinos in Diracon model. Phys.Lett., B762:162, 2016.

[70] S.Centelles et al, CP violation from flavor symmetry in a lepton quarticity dark matter model. Phys.Lett, B761:431, 2016.

[71] Y. Chikashige, Rabindra N. Mohapatra, and R.D. Peccei. Are There Real Goldstone Bosons Associated with Broken Lepton Number? Phys.Lett., B98:265-268, 1981.

[72] J. Schechter and J.W.F. Valle. Neutrino Decay and Spontaneous Violation of Lepton Number. Phys.Rev., D25:774, 1982.

[73] C.Bonilla et al. Consistency of the triplet seesaw model revisited. Phys.Rev, D92:075028, 2015

[74] C. Bonilla, R. Fonseca, and J.W.F. Valle. Vacuum stability with spontaneous violation of lepton number. Phys.Lett., B756:345-349, 2016.

[75] M. Dittmar et al. Production mechanisms and signatures of isosinglet neutral heavy leptons in $\mathrm{Z}$ decays. Nucl. Phys., B332:1, 1990.

[76] S.P. Das, et al. Heavy Neutrinos and Lepton Flavour Violation in Left-Right Symmetric Models at the LHC. Phys.Rev., D86:055006, 2012.

[77] Frank F. Deppisch, Nishita Desai, and Jose W. F. Valle. Is charged lepton flavor violation a high energy phenomenon? Phys.Rev., D89:051302, 2014.

[78] Frank F. Deppisch, P. S. Bhupal Dev, and Apostolos Pilaftsis. Neutrinos and Collider Physics. New J. Phys., 17(7):075019, 2015.

[79] K.S. Babu, Ernest Ma, and J.W.F. Valle. Underlying A(4) symmetry for the neutrino mass matrix and the quark mixing matrix. Phys.Lett., B552:207-213, 2003.

[80] S. Morisi et al. Neutrino mixing with revamped $A_{4}$ flavor symmetry. Phys.Rev., D88:016003, 2013.

[81] Sabya Chatterjee et al. Cornering the revamped BMV model ... Phys.Lett., B774:179, 2017.

[82] Peng Chen et al. Warped flavor symmetry predictions for neutrino physics. JHEP, 1601:007, 2016.

[83] Sofiane M. Boucenna et al. Small neutrino masses and gauge coupling unification. Phys.Rev., D91:031702, 2015.

[84] Frank Deppisch et al. 331 Models and Grand Unification:. Phys.Lett., B762:432, 2016.

[85] M. Reig et al. A Model of Comprehensive Unification. Phys.Lett., B774:667-670, 2017.

[86] Ernest Ma. Verifiable radiative seesaw mechanism ..Phys.Rev, D73:077301, 2006

[87] Ivania Ávila et al. Minimalistic scotogenic scalar dark matter. arXiv:1910.08422.

[88] J.W.F. Valle. Neutrino physics from A to Z: two lectures at Corfu. arXiv:1705.00872, and Neutrinos in High Energy and Astroparticle Physics Wiley-VCH (2015) textbook 